HUNGARIAN AGRICULTURAL ENGINEERING

$N^{\circ} 35 / 2019$ 59-

Published online: http://hae-journals.org/

HU ISSN 0864-7410 (Print) / HU ISSN 2415-9751(Online)

DOI: 10.17676/HAE.2019.35.59

Received: 2019.08.31. - Accepted: 2019.09.10.
PERIODICAL OF THE COMITTEE OF AGRICULTURAL AND BIOSYSTEM

ENGINEERING OF

THE HUNGARIAN ACADEMY OF SCIENCES

and

SZENT ISTVÁN UNIVERSITY

Faculty of Mechanical Engineering

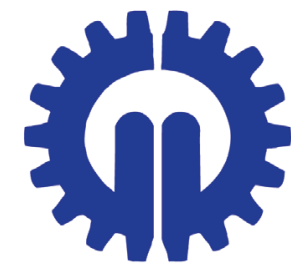

\title{
POSSIBILITIES OF USING ROBOTS IN AGRICULTURE
}

\section{Author(s):}

I. Husti

\section{Affiliation:}

Department of Applied Management, Faculty of Mechanical Engineering, Szent István University, Gödöllő, Hungary

\section{Email address:}

husti.istvan@gek.szie.hu

\section{Abstract}

The spread of robots is a global phenomenon. We can meet them today in different areas of life. Robotization has a relatively long history in industrial production and, in recent years, agricultural robots have emerged. A shrinking workforce mainly inspires the development of agricultural robots. It is a major challenge for developers to work with agriculture, as opposed to industry, with living materials and organisms. Because of these improvements, there are nowadays many successful examples of the use of robots in various agricultural operations.

\section{Keywords}

Robots, agricultural technologies, agricultural robots service organizations are increasingly being used instead of human labor for robots to perform various tasks. According to early ideas, a robot could well replace humans in a difficult situation to perform dangerous and high-precision monotone tasks.

The industrial application of robots has a relatively long history. In many countries of the world and in many areas of economic life, we can find robots that work well. They are particularly widespread in the automotive industry.

Today, it is the second biggest market for professional robotics. Estimated to be strong demand for robots throughout the farming process, including planting, pruning, weeding, pick-and-place, sorting, seeding, spraying, harvesting, and materials handling (Fig. 1.).

\section{Introduction}

The spread of robots is a global phenomenon. We meet them in many different areas of life; production-

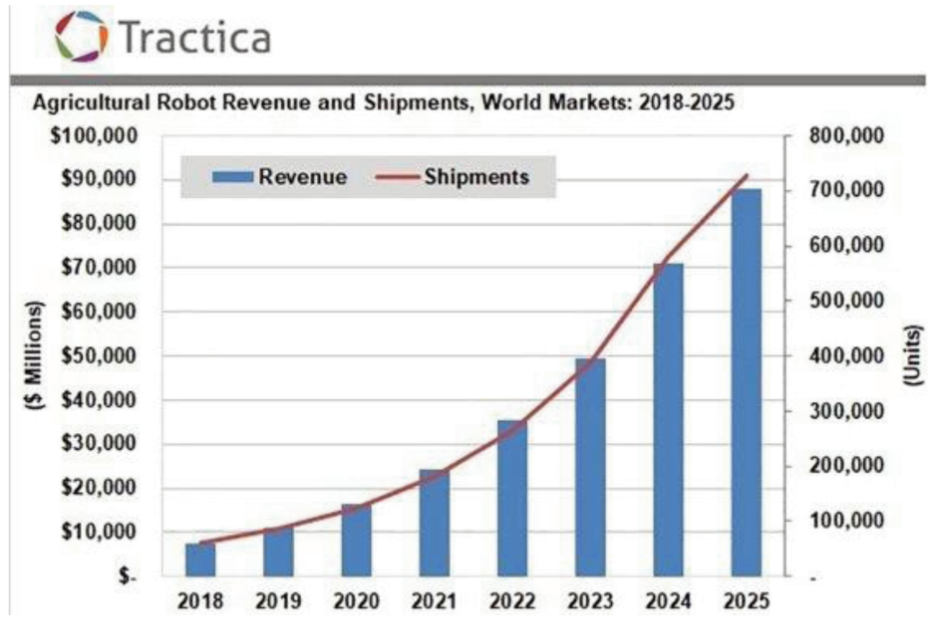

Figure 1. Agricultural Robot Revenue and Shipments (World Markets 2018-2025)

Source: (Tractica, 2016) 
Figure 1 shows the projected dynamic growth expected in the sales of agricultural robots. It's wellknown that food is a necessity that must be produced at all costs. We therefore need either more farmers or more ways to produce food with limited manpower. The robots are coming to the rescue." Within the next decade, farming as we know it is expected to be revolutionized by the use of self-driving tractors and robots that can perform time-consuming tasks now done by humans. However, more and more robots have recently been developed and tested for use in agriculture. Although some of these technologies are already available, most are at the research stage in labs and spin-off companies. Rising costs for farm labour and falling costs for self-driving technology also will provide further catalysts for the shift (Danielsca, 2016). The main purpose of this article is to demonstrate, through arbitrary examples, which areas of the robots developed as a result of improvements can be used in agriculture. A further aim is to summarize the opportunities and conditions necessary for the efficient use of robots also under the conditions of Hungarian agriculture. Nowadays robots can be found in almost every field of agriculture. Due to the limitations of the article, we are only discussing some field robots for illustration purposes.

\section{Materials and Methods}

Today, many robots can be used in agricultural technology. In accordance with the aims of the article, only a few of them are presented for purposes of illustration. Beyond personal experience, we rely on a large number of - mainly electronic - literary resources. In the author's opinion, the robots to be presented can be a good guide for domestic farmers in their technological developments.

\section{Results and Discussions}

There are some benefits of using robots. The most significant of these are following:

-The robots will be autonomous. While people will be able to focus their mind on more interesting and important tasks. Whether it'll be purveyance, removing weeds and pest, caring for crops, you won't have to be behind them to get the job done. Thanks to online databases and forecasts, combined with the data provided by external and internal sensors, they will have a complete understanding of the situation, far beyond what any human could. This will allow them to make informed decisions and obtain the optimal outcome.

-As all of these robots will be powered on batteries and electric motors, they will also have a positive impact on the energy consumption and carbon footprint of the farms.

-Some of them might even be perfectly independent thanks to solar panels on them. They'll be an important part of a greener future for mass-scale farming. Besides, this will also allow for a quieter exploitation. Thus it will also reduce the noise pollution generated by agriculture.

-The robots will help optimize the yield of the farms far beyond what could humanly be possible. Maximizing the production while minimizing the costs in energy, water, time, Tractors relentlessly roaming the fields with drones surveying cattle day and night and rovers on a permanent weed-control duty. Watering hectares and hectares of lands using only the optimal amount of water for each square meter.

-Farm employment is far from a riskless job. It often exposes workers to pesticides, long working hours in the heat and sun, and poor sanitary conditions among other direct health risks. There are many serious health hazards affecting farmworkers. For instance, the Organic Dust Toxic Syndrome, caused by grain and silage dust, is a common illness among farmers. Less known, the exposure to excessive noise produced by machines can permanently damage workers' hearing. Furthermore, most of the work is physical and outside. Thus increasing the risk of accidents caused by fatigue, difficult environment and weather, and aging tools. However, only some of these risks are being covered by employment and health insurances. By bringing robots to do these tasks, we will be reducing the risks on the human workers. Which will not only have a positive impact on their lives, but also on the people around them, and the society at large.

-And obviously, as mentioned above, the more efficient the farming, the better we all are. Maximizing yield while reducing the environmental and societal costs can help us tackle the challenges of a growing population and the lack of efficiency of modern intensive farming. We can all agree that a healthier planet would be a very strong improvement in everybody's lives!

As with any technological revolution, many questions need to be answered. Due to the fact that these robots will be in an open environment and will probably be massive pieces of machinery. While it's for obvious security reasons (you don't want a pilotless combine to go on a road trip), it can create serious entry barriers to start-up trying to break into this field (Jpvalery, 2018).

The development of agricultural robots must follow different principles than industrial robots. The reason 
for this is that agricultural production differs significantly from that of industry. In the development of agricultural robots, so-called "agricultural features" should be taken into account. The most important of them are the following:

-We work with living materials in agriculture. It follows that technological interventions require special sensitivity, unlike most industrial operations -In the context of the foregoing: technological operations can be carried out under strict timelimits in an optimal quality. These time limits are defined by "biologically-agro-technically optimal" time intervals for technological operations in crop production.

-Agricultural production is influenced by many factors. Because of this, the technological operations are very different. In addition to soil conditions and meteorological conditions, a number of other factors influence the need to perform the same technological operation differently on different fields.

-The quality of agricultural operations cannot always be assessed immediately (in real time). Consider, for example, sowing, fertilizing, or plant protection.
The quality of these can only be judged well after work.

-Agricultural work is usually carried out not in one place but in a fragmented manner. In this case, moving and transporting devices and materials between boards is a special task.

The following are some examples of the wellknown robotic solutions for growing field crops. The examples are arbitrarily changed without logical considerations. The examples illustrate what kind of robotic solutions can be used in agricultural practice.

\section{Case IH autonomous tractor}

Case IH and CNH Industrial's Innovation Group based the cables autonomous concept on an existing Case IH Magnum tractor with reimagined styling. The vehicle was built for a fully interactive interface to allow for remote monitoring of pre-programmed operations. The on-board system automatically accounts for implement widths and plots the most efficient paths depending on the terrain, obstructions and other machines in use in the same field. The remote operator can supervise and adjust pathways via a desktop computer or portable tablet interface.

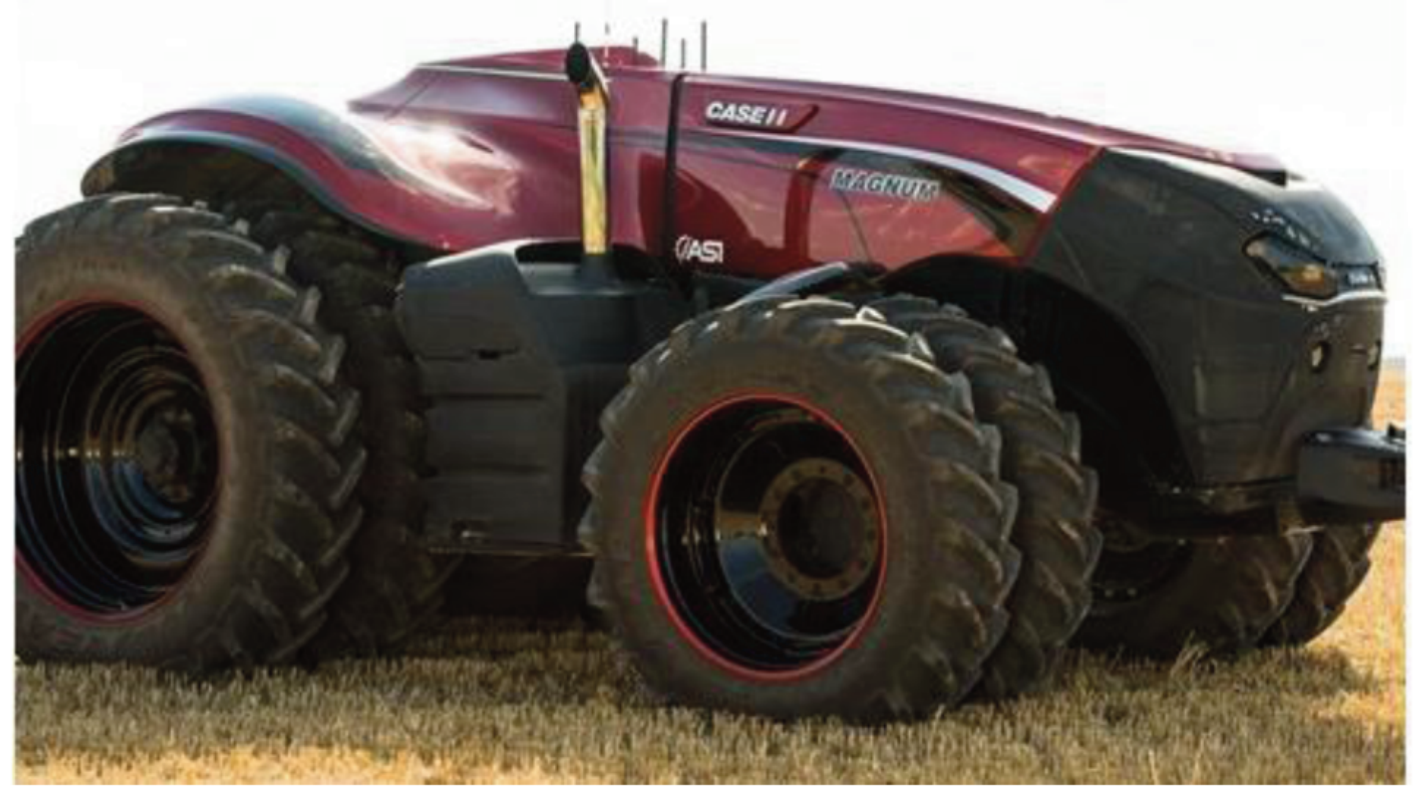

Figure 2. Case IH autonomous tractor Source: (Racine, 2016)

Through the use of radar, lidar (light imaging, detection, and ranging) and on board video cameras, the vehicle can sense stationary or moving obstacles in its path and will stop on its own until the operator, notified by audio and visual alerts, assigns a new path. The vehicle will also stop immediately if GPS signal or position data is lost, or if the manual stop button is pushed. Machine tasks can also be modified in real time with via remote interface or automatic weather warnings (Racine, 2016).

"In many parts of the world, finding skilled labor during peak use seasons is a constant challenge for 
our customers," said Case IH Brand President Andreas Klauser."Whileweoffer auto-steering and telematics on our equipment today for remote management of farm machinery and employees, this autonomous tractor concept demonstrates how our customers and their employees could remotely monitor and control machines directly. This technology will offer our customers greater operational efficiencies for tasks such as tillage, planting, spraying and harvesting." (Racine, 2016).

\section{Rowbot}

Minneapolis-based start-up company is trying to bring more automation into agriculture. It has invented a self-driving "rowbot" that's basically a lightweight tank on wheels with sensors and a GPS system that can drive between row crops, dispensing nitrogen fertilizer or completing other tasks. "Rowbots" can fertilize crops more efficiently and precisely, and hopefully in the future, plant some seeds.
Normally a corn farmer will spray much of the nitrogen fertilizer at the time of planting, and sidedress the corn crop with additional nitrogen in midJune before stalks grow too high.

The problem is that spring rains often wash away much of the nitrogen, which isn't really needed the most until the plant is growing fastest in June and July. Even adding nitrogen in June can be tricky, said Cavender-Bares, because heavy equipment can cause ruts, and by July the cornstalks may be too high to allow spraying.

The idea is for a fleet of "rowbots" to drive through a field two or three times a summer when the corn is growing most rapidly, spraying fertilizer to cover four rows at a time in the places and amounts where it is needed. The robots can also be linked to systems that tell it how much fertilizer to apply to different parts of the field, thereby saving farmers time and money (Meersman, 2018).

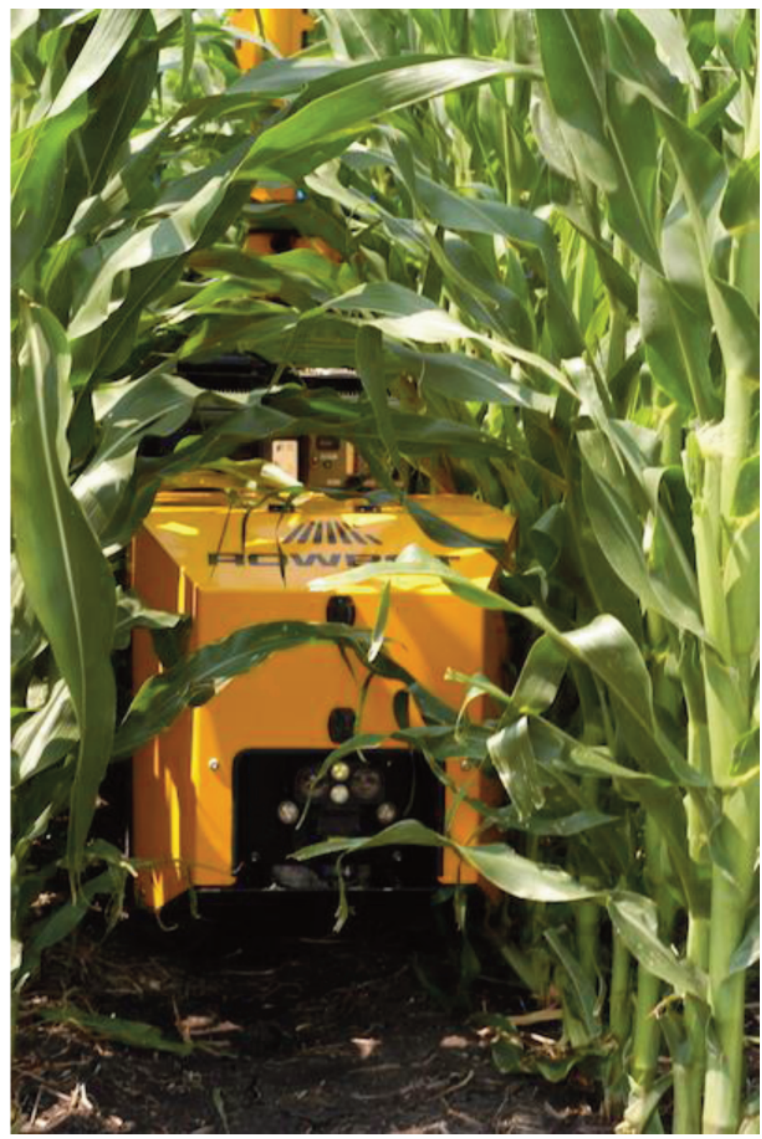

Figure 3. Rowbot in action Source: (Rosen, 2014)

Small enough to easily travel between planted rows of corn, Rowbot reduces the amount of fertilizer farmers use by dispensing it at the right time in the right amounts. The timing for corn is critical because once corn plants get too tall to run a tractor through the field farmers may miss applying fertilizer when it can be most beneficial. But with Rowbot the plants are undisturbed because of its small form factor. And because it provides the nitrogen directly in what is referred to as "side dressing" it eliminates potential 
overfeeding and nitrogen runoff which leads to polluting of local ponds, rivers and lakes. Thanks to its adjustable width $(1.4 \mathrm{~m}$ to $2.1 \mathrm{~m})$ and the positioning of the adjustable sensor ( $1 \mathrm{~m}$ to $1.8 \mathrm{~m})$, the robot can be used on a wide variety of crops, for example cereals, beets, market garden crops (Rosen, 2014).

\section{BoniRob}

Given the scale of farming today, treating weeds chemically is really the only practical way for humans to keep them under control, because you can use tractors or airplanes to cover large areas in a short amount of time. But all of those necessarily deadly (to weeds) chemicals then get on the plants we don't want to kill (because we want to eat them), as well as getting washed into the soil. The most organic and eco-friendly way of dealing with weeds is the oldfashioned way: physically removing them. "Physical removal" can mean pulling weeds out completely, but that involves both grasping the weed and doing something with it. A better solution is to just smash it way down into the ground, which is faster, easier, and something a robot can do excellently.

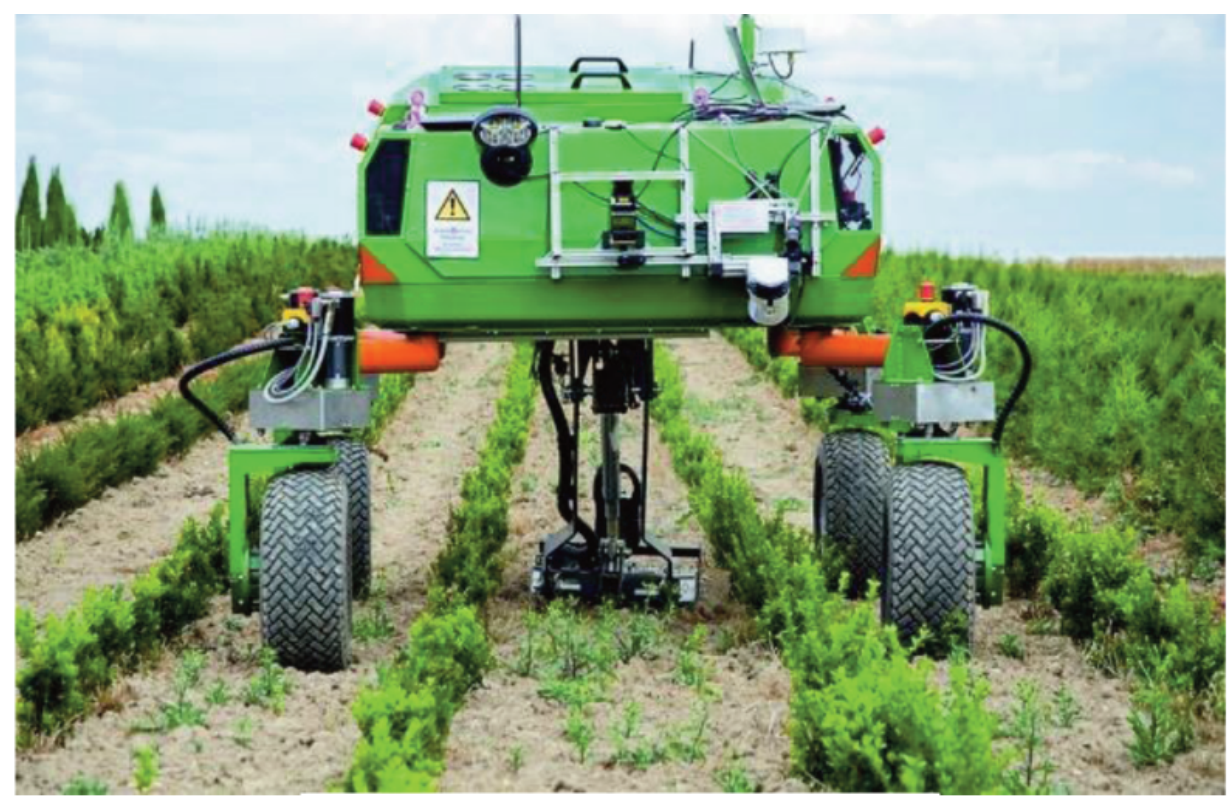

Figure 4. The BoniRob Source: (Ackerman, 2015)

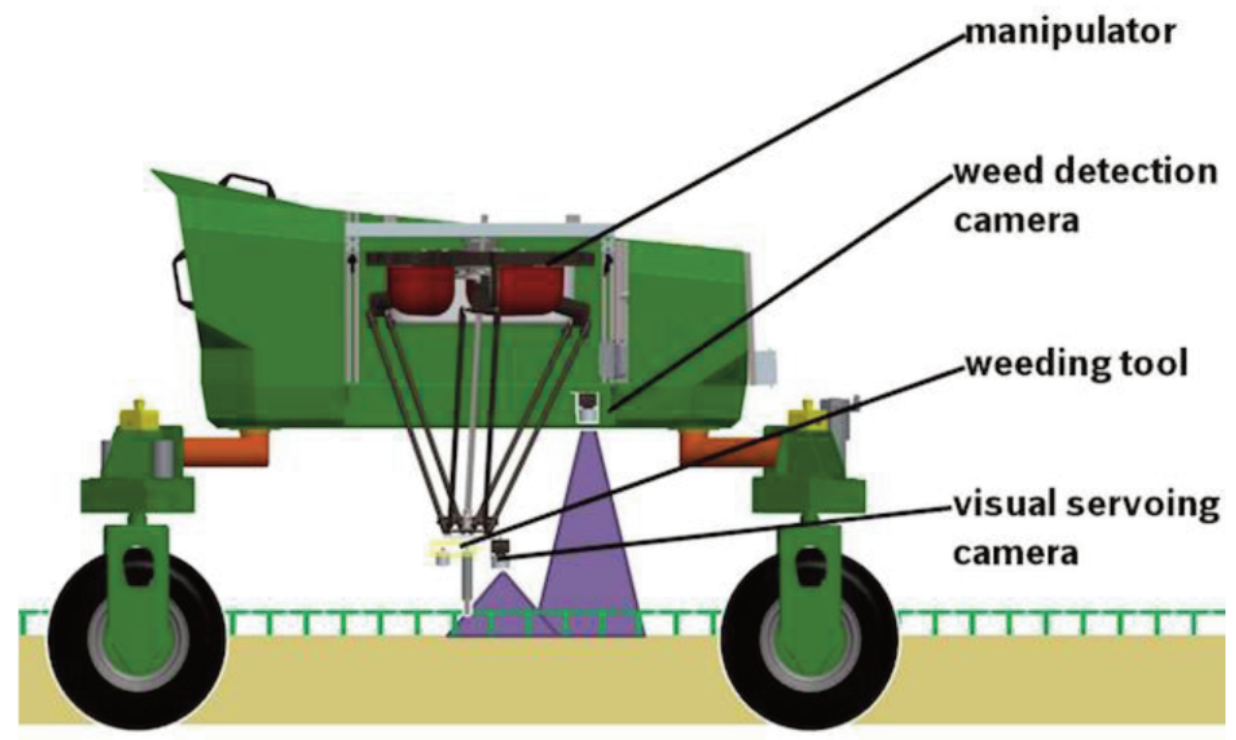

Figure 5. The main elments of BoniRob Source: (DIYI Team, 2015) 
The stamping tool is 1 centimetre wide, and it drives weeds about $3 \mathrm{~cm}$ into the soil. It's designed to detect (through leaf shape) and destroy small weeds that have just sprouted, although for larger weeds, it can hammer them multiple times in a row with a cycle time of under $100 \mathrm{~ms}$. Testing on a real carrot crop, which has carrots spaced about $2 \mathrm{~cm}$ apart and an average of 20 weeds per meter growing very close to the carrots themselves, the robot had no trouble at all. The maximum capability of the system is about 1.75 weeds per second at a speed of $3.7 \mathrm{~cm} / \mathrm{s}$ and a weed density of 43 weeds per meter, but at lower weed densities, the speed can be cranked up to $9 \mathrm{~cm} / \mathrm{s}$.

BoniRob can navigate itself, adapting to many different field configurations. Its modular payload bay can handle up to 150 kilograms of stuff, and an onboard generator lets it run autonomously for
24 hours without needing to refuel (Ackerman, 2015)

\section{Ecorobotix}

Targeted application of herbicide by the first ever completely autonomous robot for the ecological and economical weeding of row crops, meadows and intercropping cultures. The robot works without being controlled by a human operator. It covers the ground just by getting its bearings and positioning itself with the help of its camera and GPS. Its system of vision enables it to follow crop rows, and to detect the presence and position of weeds in and between the rows. Two robotic arms then apply a micro dose of herbicide, systematically targeting the weeds that have been detected. In bare fields or meadows the robot positions itself precisely thanks to its GPS RTK.

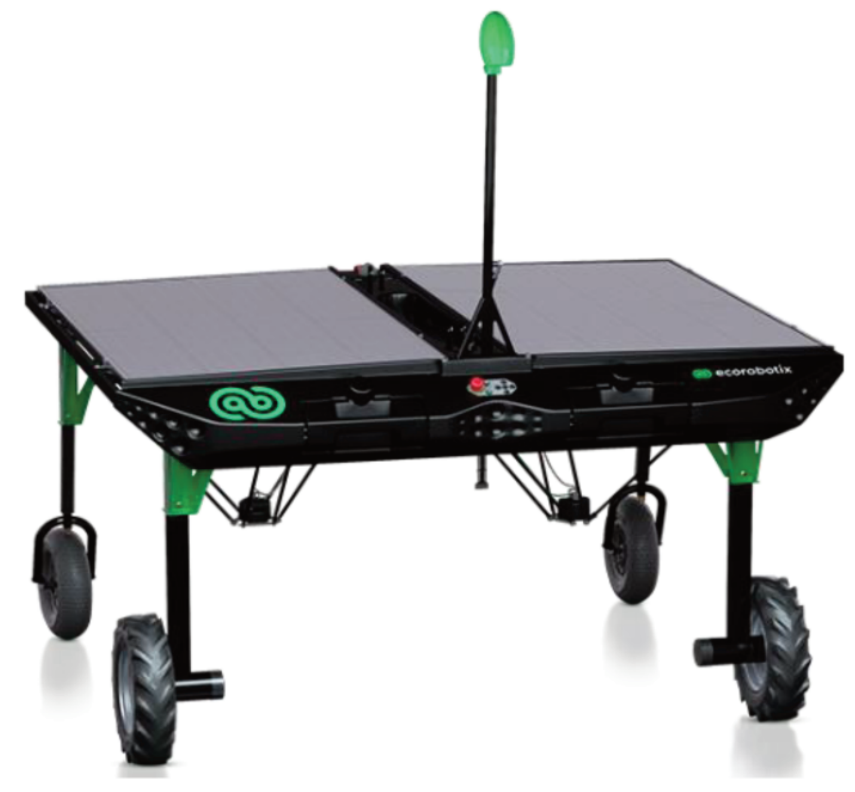

Figure 6. Ecorobotix with photovoltaic panels Source: (Ecorobotix, 2019)

Reliance on solar power makes the robot completely autonomous in terms of energy, even when the weather is overcast. As it adapts its speed to the concentration of weeds, it is most suitable for use in fields where the level of concentration is low to moderate, in order to cover the ground at a reasonable speed. We recommend using the machine after an initial standard application of herbicide, in order to replace subsequent applications and thus save an important amount of herbicide (more than 20x less than with a standard treatment). The machine can be completely controlled and configured by means of a Smartphone app (Ecorobotix, 2019).

\section{SwarmFarm Robotics Philosophy}

SwarmFarm never set out to automate agriculture or save labour - rather, their philosophy is to create new farming systems that are not possible on the back of a tractor.

The company was founded by farmers who have spent 20 years farming with modern technology that has seen such equipment as tractors, sprayers and planting machinery become increasingly larger and more complex. The farmers came up with the idea that multiples of small, lightweight robots working together in a 'swarm' would be the driver of new AgTech into farming. 
A world-first robot designed to work with others to spray crops in swarms, and ultimately help to fill the rural labour shortage, SwarmFarm Robotics develops robots for farmers using advanced technologies to create new field practices and farming methods that simply aren't possible through traditional farming methods. For farmers, this means increased efficiency, higher yields and a lower impact on the environment. Swarm robots - small, simple and inexpensive - are poised to reverse the trend in agriculture towards ever bigger machines. These little workers behave autonomously, interacting with each other and the environment to achieve the desired outcome. The benefits include less damage to soil structure and greater precision for weeding and harvesting.

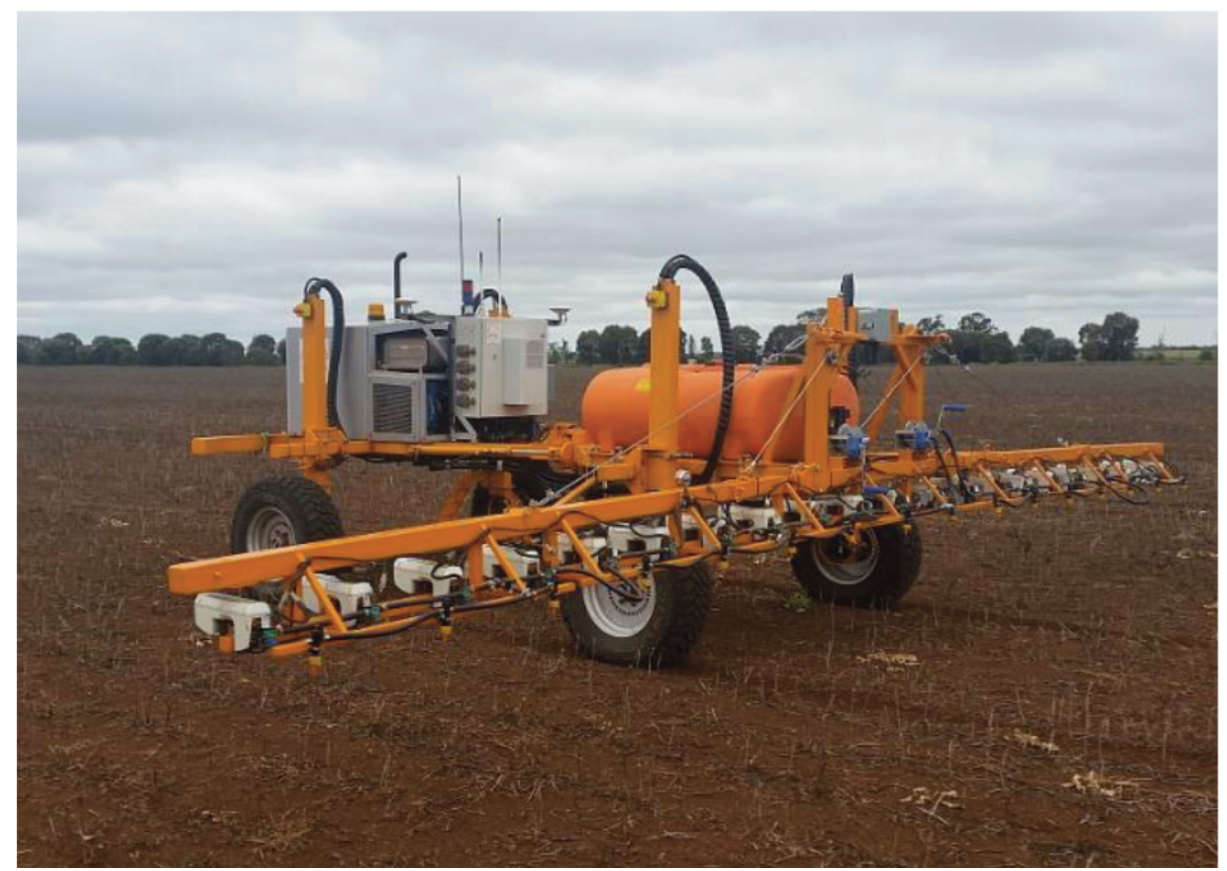

Figure 7. 4WD SwarmFarm robots carrying WeedSeeker technology cover the paddock spraying only living weeds Source: (Bloomer, 2017)

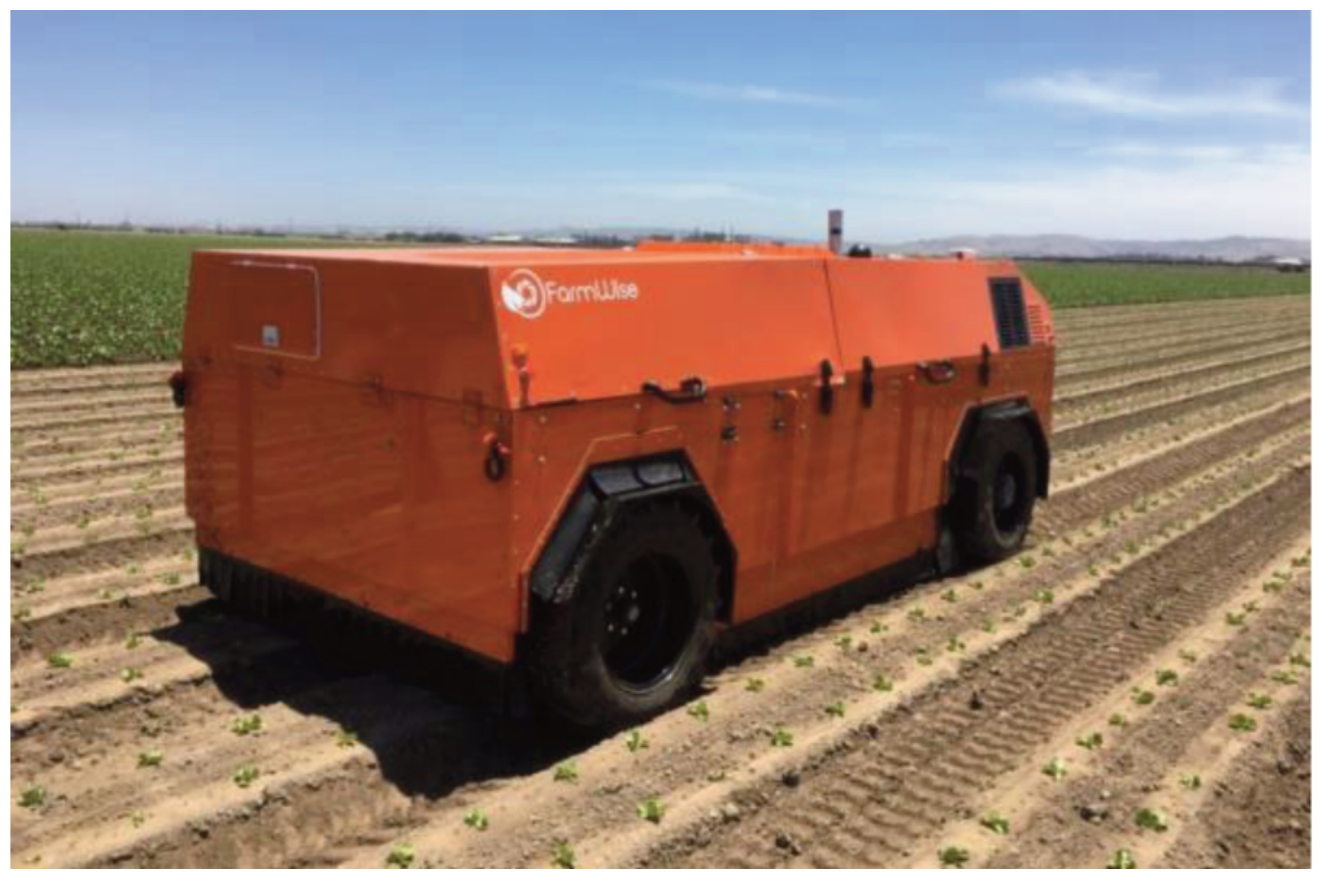

Figure 8. A robotic weeder leaves lettuce crops unharmed

Source: (FarmWise,2019) 


\section{FarmWise vegetable weeder}

Silicon Valley start-up FarmWise ${ }^{1}$ was founded in 2016 to create adaptable, robotic machinery that can help farmers improve productivity, crop health, and yields - thereby making agriculture more efficient and profitable. Also important to FarmWise is leveraging artificial intelligence technology to reduce the amount of herbicides and weed-killing chemicals farmers use. In the future, FarmWise would like to expand its artificial intelligence platform to automate other agricultural tasks, such as watering fields and harvesting crops.

The FarmWise machine drives over rows, detects weeds in the soil, cuts their roots and tosses them out of the row with an action mimicking that of a person using a hoe. Currently, one of the machines is operated in part by an on-field operator and a "teleoperation service" from a central centre. But the company hopes its machines will reach a higher level of autonomy by early 2019 so that one on-field operator could supervise more than one of the machines.

FarmWise's robots use a perception system powered by deep learning to capture and analyse plant images in real-time through the latest and finest available embedded computers.

Once the images are processed, the machines know where the weeds are and mechanically act on each plant: removing weeds around them if encountered without using a single drop of herbicide.

\section{Conclusions}

The main conclusion of this article is that farmers can already choose from several robotic options for improving agricultural technology and some of them already available. Rising costs for farm labor and expected falling costs for robotic technology also will provide further catalysts for the shift.

Although robots are about tomorrow, we must prepare for them today. Two interesting questions that remain are: Are the robots ready for farmers? Are farmers ready for the robots? These questions also need to be answered in Hungary. Not to mention that there are no universal solutions. There are no two identical farms, but there are two identical robots. Therefore, every farmer needs to think about whether he is taking advantage of robots or staying with conventional farming.

In this paper, we do not discuss the economic issues of using robots. A detailed examination of this topic is a task for the future.

\section{References}

[1] Ackerman, E. (2015). Bosch 's Giant Robot Can Punch Weeds to Death. IEEE Spectrum, 0-1. Retrieved from https://spectrum.ieee.org/automaton/robotics/industri al-robots/bosch-deepfield-robotics-weed-control

[2.] Alexander, D. (2018): 9 Robots That Are Invading the Agriculture Industry

https://interestingengineering.com/9-robots-that-areinvading-the-agriculture-industry

https://blog.robotiq.com/top-10-robotic-applicationsin-the-agricultural-industry

[3.] Artificial Intelligence Revenue to Reach $\$ 36.8$ Billion Worldwide by 2025 .

https://www.tractica.com/newsroom/pressreleases/artificial-intelligence-revenue-to-reach-368-billion-worldwide-by-2025/

[4.] Bloomer, D. (2017). In Search of Farm Robots: Ch $1 \mid$ LandWISE. Retrieved October 17, 2019, from http://www.landwise.org.nz/precision-agriculture/insearch-of-farm-robots-ch-1/

[5.] Claver, H. (2019). Agricultural robot shipments to increase sharply - FutureFarming. Retrieved September 13, 2019, from

https:/www.futurefarming.com/Machinery/Articles/2 019/7/Agricultural-robot-shipments-to-increase$\mathrm{sh}$ arp $1 \mathrm{y}-455361 \mathrm{E} /$ ? in t c m p = relatedcontent\&intcmp $=$ related-content

[6.] Danielsca, J. (2016). Future of farming: driverless tractors, ag robots. Retrieved October 17, 2019, from https://www.cnbc.com/2016/09/16/futureof-farming-driverless-tractors-ag-robots.html

[7.] DIYI Team. (2015). BoniRob - Vision-Based High-Speed Manipulation for Robotic Ultra-Precise Weed Control - Do It Yourself India Magazine. Retrieved October 17, 2019, from https://diyin.wordpress.com/2015/12/15/bonirobvision-based-high-speed-manipulation-for-roboticultra-precise-weed-control/

[8.] Ecorobotix. (n.d.). Technology fir environment: an innovative, autonomous and economical machine. Retrieved from https://www.ecorobotix.com/wpcontent/uploads/2017/02/ECOX_FlyerPres 18-EN1_RVB-1.pdf

[9.] Ford, M. (2015) Rise of the robots. Basic Books, New York. http://digamo.free.fr/marford15.pdf [10.] Jpvalery. (2018). Is the Future of Agriculture in Robotics? Retrieved September 10, 2019, from https://www.robotshop.com/community/blog/show/is -the-future-of-agriculture-in-robotics

[11.] Lowenberg-DeBoer, J. et al. (2019): Economics of robots and automation in field crop production. 
Vol.:(0123456789) Precision Agriculturehttps: //doi.org/10.1007/s11119-019-09667-5

https://link.springer.com/content/pdf/10.1007\%2Fs11 119-019-09667-5.pdf

[12.] Meersman, T. (2018). Rowbots Systems help bring the future to farming - StarTribune.com. Retrieved September 7, 2019, from http://www.startribune.com/rowbots-systems-helpbring-the-future-to-farming/478998163/

[13.] Oliver, J. (2019): Future of agricultural mechanisation seems promising

https://www.futurefarming.com/Machinery/Articles/2 019/10/Future-of-agricultural-mechanisation-seemspromising-485287E/

[14.] Racine, W. (2018). CNH Industrial Newsroom : Case IH Premieres Concept Vehicle at Farm Progress Show. Retrieved June 14, 2018, from https://media.cnhindustrial.com/EUROPE/CASEIH/case-ih-premieres-concept-vehicle-at-farmprogress-show/s/3a2abb2b-d8a5-4b46-90bd-e788052 f7be3

[15] Roldán, JJ. et al. (2016): Robots in Agriculture: State of Art and Practical Experiences DOI: 10.5772/intechopen.69874

https://www.intechopen.com/books/servicerobots/robots-in-agriculture-state-of-art-andpractical-experiences
[16.] Rosen, L. (2014). Rowbot Robot Tends to Farmers' Fields. Retrieved August 21, 2019, from https:/hplusmagazine.com/2014/09/22/rowbot-robottends-farmers-fields/

[17.] Sennaar K. (2019): Agricultural Robots Present and Future Applications

https://emerj.com/ai-sector-overviews/agriculturalrobots-present-future-applications/

[18.] Tholhuijsen, L. (2017): How farmers will benefit from smart machinery?

https://www.futurefarming.com/Machinery/Articles/2 017/4/How-farmers-will-benefit-from-smartmachinery-459WP/

[19.] Tholhuijsen, L. (2019): Self-learning algorithm gets better at weed detection.

https://www.futurefarming.com/Machinery/Articles/2 019/10/Self-learning-algorithm-gets-better-at-weeddetection-487567E/

[20.] Yaghoubi, S. (2013): Autonomous Robotsfor Agricultural Tasks and Farm Assignment and Future Trends in Agro Robots.International Journal of Mechanical \& Mechatronics Engineering IJMMEIJENS Vol:13 No:03 https://citeseerx.ist.psu.edu/viewdoc/download?doi= 10.1.1.418.3615\&rep=rep1\&type $=$ pdf 